\title{
Liderazgo en enfermería: ¡Es hora de dar un paso al frente!
}

\section{Leadership in Nursing: Time to Step Up!}

\section{Liderança em enfermagem, é hora de dar um passo a frente!}

El año pasado se presentaron varios hitos históricos que pedían fortalecer la capacidad de liderazgo, el desarrollo y las oportunidades para la enfermería en todo el mundo. El informe de la Organización Mundial de la Salud (OMS) titulado Situación de la enfermería en el mundo 2020: invertir en educación, empleo y liderazgo reveló que sólo 53\% ( $\mathrm{n}=78)$ de los países tenían un programa nacional de desarrollo de liderazgo, de los cuales la mayoría se encontraba en las regiones de África y el Este del Mediterráneo1.

Dentro del informe de la Organización Panamericana de la Salud (OPS) titulado Orientación estratégica para enfermería en la Región de las Américas, una de las principales recomendaciones identificadas fue fortalecer y consolidar el liderazgo y la gestión estratégica de la enfermería en el contexto de los sistemas de salud para la formulación de políticas y seguimiento².

Las estrategias para avanzar en este objetivo incluyen el fortalecimiento de la capacidad de las enfermeras para trabajar en colaboración con múltiples socios para impulsar un cambio positivo y sostenible en los sistemas de salud; mejorar la calidad de la educación en enfermería con el fin de responder a las necesidades de los sistemas de salud en el camino hacia el acceso y cobertura universal de la salud, así como los objetivos estratégicos de desarrollo y promover cursos de capacitación en liderazgo y gestión a través de programas de educación continua. Si bien para algunos estos pueden parecer objetivos aspiracionales elevados, el liderazgo en enfermería es algo a lo que todos debemos prestar atención, en todos los niveles y en todos los entornos.

El liderazgo se puede definir como la actividad de conducir a un grupo de personas o a una organización con miras a establecer una visión clara que pueda difundirse a otros para que la sigan voluntariamente y proporcionen información, conocimiento y métodos para su realización, y coordinen y equilibren los intereses en conflicto de todos los miembros e interesados directos ${ }^{2}$.

Los rasgos comunes de los líderes comprenden un fuerte carácter moral, humildad y coraje. Las habilidades asociadas con el liderazgo que se pueden desarrollar incluyen la comunicación, la delegación y la creación de una visión. El famoso entrenador de fútbol americano Vince Lombardi expresó 
que los líderes se hacen, no nacen. Se hacen con mucho esfuerzo... para lograr cualquier objetivo que valga la pena. Esta cita resume las concepciones de que los líderes pueden tener rasgos innatos, pero también desarrollan habilidades que son moldeadas por experiencias, por lo tanto, podemos decir que se pueden desarrollar líderes.

Entonces, ¿por qué es tan importante desarrollar el liderazgo entre las enfermeras hoy en día? Primero, debe reconocerse que las enfermeras SON líderes, ya sea en la cabecera de la cama, en la sala de juntas o en el gobierno. Los profesionales de enfermería son expertos en valorar una situación de manera integral, identificar el problema y los enfoques con los pacientes, las familias y las comunidades, implementar y evaluar los resultados y reajustar según sea necesario.

Las y los enfermeros aportan conocimientos importantes sobre las decisiones de atención del paciente, ya que ayudan a las familias a tomar resoluciones que les permitan gestionar su salud en el contexto de sus vidas. Son profesionales expertos en comprender el impacto del trabajo, la escuela, la vida en el hogar y otros determinantes sociales de la salud y cuál es la mejor manera de desplazarse por los servicios de salud para apoyar los objetivos del paciente y la familia. A nivel de gestión y políticas, las enfermeras son defensoras que amplifican la voz de los pacientes así como de sus familias en la toma de decisiones. Dado el importante papel que desempeñan, es necesario preparar y brindar oportunidades para que más enfermeras lideren las mejoras en la calidad, la seguridad, el acceso y el valor de la atención médica.

La pregunta para todas las enfermeras es: ¿cuáles son las estrategias para desarrollar su propio potencial de liderazgo? Comparto con ustedes las lecciones aprendidas en mi viaje de liderazgo en una carrera que abarca posiciones como: enfermera asistencial, gerente de enfermería, investigadora de enfermería y, en última instancia, en mi función ahora como decana de enfermería en la Universidad de Pensilvania. Un punto de partida para su viaje de liderazgo es preguntarse: ¿cuáles son mis fortalezas de liderazgo?, ¿qué habilidades de liderazgo son importantes para mí para ser el tipo de líder que aspiro a ser? Estas preguntas afirman que todos tenemos fortalezas y características de liderazgo sobre las que podemos construir, al mismo tiempo que reconocemos que hay habilidades que podemos desarrollar.

Basado en el conocimiento experiencial obtenido de las interacciones con enfermeras líderes, desarrollé un marco para el liderazgo latino en enfermería que considera rasgos específicos (integridad, visión, conocimiento profesional, habilidades, expertise e inteligencia emocional), un proceso en el que se desarrollan más conocimientos y habilidades, así como cualidades integrales para trabajar con comunidades latinas en los Estados Unidos (por ejemplo, conocimiento cultural latino, conciencia, habilidades, conexión con las comunidades)33. Este marco me ha servido de guía para mi desarrollo personal y profesional, el cual se ha modificado paulatinamente con el tiempo.

En segundo lugar, otra estrategia importante para el incremento del liderazgo es desarrollar una visión de lo que quieren cumplir en su carrera. Esta visión puede ser a corto plazo (por ejemplo, quiero ser un gerente de enfermería) o a largo plazo (por ejemplo, quiero ser un ejecutivo del sistema de salud). Una vez que se tenga una visión, el siguiente paso es desarrollar un plan sobre cómollegar ahí. Uno de mis objetivos a corto plazo al principio de mi carrera fue publicar en una revista de enfermería. Pensé que al realizarlo sería una forma de tener un mayor impacto en la práctica clínica. Traté de escribir, por mí misma, algunos artículos de base clínica, pero fueron rechazados. Me involucré en la investigación principalmente como un medio para publicar y ampliar mi impacto. Publiqué, pero lo más importante es que mi participación en la investigación me llevó a realizar un doctorado. La lección que aprendí de esto es que el fracaso en trabajar por mi cuenta y mi continua determinación de alcanzar una meta a corto plazo me llevaron a oportunidades que no había considerado. 
Al crear su visión y plan es importante que establezcan metas que los mantengan enfocados en las posibilidades. Es fundamental tomar medidas para respaldar sus metas, construir el tiempo y la energía que les permitan decir sí a las oportunidades que los llevarán a alcanzar sus objetivos. Esto significa evaluar y buscar oportunidades que respalden mejor su éxito. Como muchos, a menudo me he enfrentado a desafíos que no han sido fáciles, o me he mostrado reacia a enfrentar retos que no quería hacer por una variedad de razones. Una vez que acepté el desafío, dediqué tiempo y esfuerzo para asegurarme de que éste se pudiera completar con éxito. Estas experiencias siempre han tenido beneficios que han contribuido a mi crecimiento personal y profesional.

En tercer lugar, otro aspecto del desarrollo del liderazgo es encontrar el entorno adecuado, ya sea en el trabajo o en la escuela, que sea colaborativo y se centre en su éxito. He tenido la suerte de haber trabajado en equipos de investigación que dejaron en claro que estaban comprometidos con mi éxito. Si se encuentran en un entorno que no respalda sus objetivos, necesitan encontrar el coraje para alejarse de estos, así como de las relaciones. También cambié de posición algunas veces porque sabía que tendría un mayor potencial de crecimiento.

Por ejemplo, una vez que completé mi formación posdoctoral tuve la oportunidad de seguir trabajando como directora de investigación en un hospital infantil donde había trabajado por un periodo largo; también trabajé en un grupo de política hispana con el que había estado involucrada durante años, además de laborar en escuelas importantes de enfermería en investigación. Si bien había algo que me encantaba en todas mis opciones, elegí como mi cita académica inicial la Universidad de Pensilvania. Creía que el entorno en la escuela sería el mejor para ayudar a iniciar una carrera de investigación, iy tenía razón! Significó sacrificios personales para mí y mi familia, ya que viajé de Pensilvania a Michigan durante cinco años; fue una decisión difícil, pero acertada. Tuve que hacer otros cambios en mi carrera y, aunque fue complejo en ese momento, fue lo mejor para mi desarrollo personal y profesional.

En mi propio desarrollo de liderazgo he llegado a creer que las opciones están limitadas por los propios límites que uno crea para sí mismo. Creo que el fracaso es un estado de ánimo, pero el éxito es un estado de acción. Intento no centrarme en el fracaso, pero ¿cuáles son las lecciones que se pueden aprender?, ¿qué es lo peor que me puede pasar si fallo? Si el objetivo y la tarea parecen insuperables, desglósenlos y procedan paso a paso. Cuando decidí seguir mi doctorado tenía muchas dudas sobre mi capacidad para tener éxito en el programa. Había visto fracasar a muchos de mis colegas, a quienes consideraba más inteligentes que yo. Decidí que era peor que fallar, no intentarlo y preguntarme ¿y si...? Así que lo hice, una clase a la vez, un proyecto a la vez, siempre diciéndome a mí misma que si no podía tener éxito o si decidía que un doctorado no era el camino para mí, podía cambiar de opinión.

En resumen, el mundo necesita enfermeras que dirijan jen todas partes! Es nuestra responsabilidad comprender nuestras propias habilidades de liderazgo y desarrollar habilidades que nos permitan a todos ejercer nuestro máximo potencial de liderazgo. ¡Nuestros pacientes, familias y comunidades dependen de nosotros!

A.M. Villarruel ORCID: $0000-0002-2367-5786$ Dean University of Pennsylvania, School of Nursing Philadelphia, PA, USA nursingdean@nursing.upenn.edu 


\section{REFERENCIAS}

1. World Health Organization. State of the world's nursing 2020. Investing in education, jobs, and leadership. Geneva: WHO; 2020. https://bit.ly/zaIjioU

2. Pan American Health Organization. Strategic directions for nursing in the Region of the Americas. Washington, D.C.: PAHO; 2019. https://bit.ly/2PqzCmG

3. Villarruel AM. A framework for latino nursing leadership. Nurs Sci O. 2017; 30(4): 347-52. https://doi.org/10.1177/0894318417724476 